\title{
A NEW PERSPECTIVE FOR LITHOLYSIS? INFLUENCE OF AMINES ON THE SOLUBILITY OF KIDNEY STONES IN VITRO
}

\author{
N.M. Bogdan ${ }^{1}$, V.M. Bilobrov², A.G. Matvienko²
}

\author{
${ }^{1}$ Institute of Organic Chemistry of National Academy of Sciences of Ukraine, 5, \\ Murmanska St., 02660 Kyiv, Ukraine; bohdan@ioch.kiev.ua \\ ${ }^{2}$ L.M. Litvinenko Institute of Physical Organic and Coal Chemistry, National Academy of \\ Sciences of the Ukraine, 50 Kharkivske shosse str. 02160 Kyiv, Ukraine
}

\begin{abstract}
It was shown that amines increase the equilibrium solubility of uric acid kidney stones substantially and stabilize the process of litholysis in due course. However, these amines compete with ammonium in salt formation. Imidazole and triethanolamine are stabilize of uric acid solubility when $\left[\mathrm{NH}_{4}{ }^{+}\right]<20 \mathrm{mM}$, and piperazine promotes the dissolution of uric acid if $\left[\mathrm{NH}_{4}{ }^{+}\right]>46 \mathrm{mM}$. In salt solutions simulating complete cationic composition of human urine, solubility of uric acid is practically the same for the systems with imidazole and piperazine and it is some lower for triethanolamine containing system.
\end{abstract}

Keywords: urate kidney stone, equilibrium solubility, amines.

Introduction. In our previous report we studied the influence of some anions and cations ([Cat $\left.{ }^{\mathrm{n}}\right]$ ) (ammonium, sodium, potassium, calcium and magnesium) on the solubility (S) of uric acid (HUr) stones [1]. It was shown that, in water solutions, equilibrium solubility of uric acid stones in greater extent depends on the concentration of above mentioned cations. Moreover, the maximum solubility of stones occurs at the values where concentration of above mentioned alkaline and alkaline-earth metal ions are equal to their "norm" in the urine of healthy persons.

According to our own observations and literature experimental data [2] on equilibrium solubility of various uric acid salts are quite different. It gave us ground to expect, that varying of the concentration of cations, having various "hardness" (according to Drago and Pearson) [3], in solution will enable us to change the solubility of HUr concrements. According to Drago, Hansch, and Pearson uric acid is regarded as 
moderately strong $\left(\mathrm{pK}_{\mathrm{a}}=5.45\right)$ and comparatively soft acid. For this reason, solubility of the products of its interaction with bases of various hardness must differ significantly. In the series of monovalent cations one can expect maximal solubility of uric acid salt of cesium-ion, in series of trivalent cations - for salt of cerium (Ce) -ion. Indeed, this was confirmed experimentally, though observed effect seemed to be not so great.

It should be searched for more "softer" bases among nitrogen-bearing organic compounds- amines. So, the aim of present work was to study equilibrium solubility of uric acid stones under the influence of some amines and compare it with the least soluble ammonium salt (Material and methods for elaboration of equilibrium solubility and rate of dissolution of uric acid stones, used in this work were described in details in our previous issue of this series.)

Results and discussion. Equilibrium solubility of uric acid stones $\left(\mathrm{S}_{\mathrm{HUr}}\right)$ was studied in the presence of nitrogen-bearing compounds of different classes and compared with the solubility of the least soluble ammonium salts of uric acid (see Table).

Table. Solubility of Uric Acid Stones $\left(\mathrm{S}_{\mathrm{HUr}}\right)$ in water and in $0.35 \mathrm{M}$ water-solution of amines $\left(\mathrm{pH}=8,37^{\circ} \mathrm{C}\right)$

\begin{tabular}{|c|c|c|c|}
\hline No & Compound & $\mathrm{pKa}$ & $\mathrm{S}_{\mathrm{HUr}} \cdot 10^{-4}, \mathrm{M}$ \\
\hline 1 & Water & & 3.8 \\
\hline 2 & Ammonium & 9.3 & 0.8 \\
\hline 3 & Methylamine & 10.6 & 6.2 \\
\hline 4 & Diethylamine & 11.0 & 3.0 \\
\hline 5 & Tetraethylammonium hydroxide & 14.0 & 4.9 \\
\hline 6 & Tris(hydroxymethyl)-Aminomethane & 8.1 & 8.8 \\
\hline \multirow[t]{2}{*}{7} & Piperazine & 9.8 & 13.0 \\
\hline & $(2)$ & 5.6 & \\
\hline \multirow[t]{2}{*}{8} & 3-Dimethylamino-1-propylamine (1) & 10.8 & 23.0 \\
\hline & $(2)$ & 8.2 & \\
\hline 9 & N,N,N',N'-Tetramethyl-ethylendiamine & & 25.0 \\
\hline 10 & Triethanolamine & 7.8 & 44.0 \\
\hline 11 & Imidazole & 7.0 & 57.0 \\
\hline
\end{tabular}


From the Table clearly notable that adding of ammonium-ion into water solutions leads to essential decrease of solubility of HUr calculi, while on the contrary, adding of amines leads to increase of $\mathrm{S}_{\mathrm{HUr}}$.

Nitrogen bases were divided into two groups according to the change of $\mathrm{S}_{\mathrm{HUr}}$. Compounds 3- 5 were united in the first group, they do not increase $\mathrm{S}_{\mathrm{HUr}}$ sufficiently. Application of compounds 6-11 (2nd group), causes the increase of $\mathrm{S}_{\mathrm{HUr}}$ more significantly. We did not find any quantitative correlation between $\mathrm{S}_{\mathrm{HUr}}$ and tabulated parameters of structure and reactivity of amines. However, it may be supposed that the first group contains "hard" bases, and the second one contains "soft" bases, and molecules of latter are characterized by high polarizability (molecular refraction).

Let us consider in greater detail the influence of piperazine (PiPe), triethanolamine (TEA), and imidazole (Im) on the solubility of uric acid calculi. Needed to note that, these compounds of the second group are widely used in medical practice.

In Figure 1 the dependence of $S_{\mathrm{HUr}}$ on the concentration of these amines [Am] is shown. Easily notable that the dependence $\mathrm{S}_{\mathrm{HU}}=\mathrm{f}([\mathrm{Am}])$ differ clearly, and at $[\mathrm{Am}]=0.35 \mathrm{M}$ solubility of uric acid has a linear dependence on pKa of amines (fig. 2). More significantly the differences are provided, when $\mathrm{pH}$ values of solutions are varied (fig. 3).

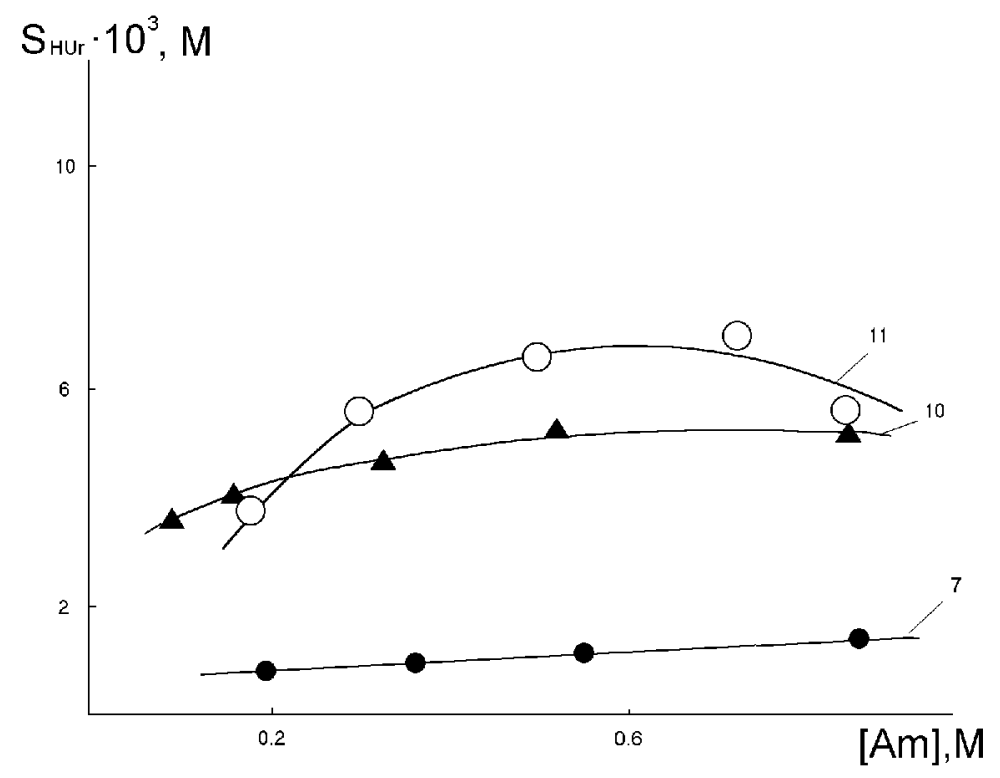

Figure 1. Dependence of equilibrium solubility of uric acid stones on the concentration of amines. The numbers of compounds in the figure and in the Table are the same 


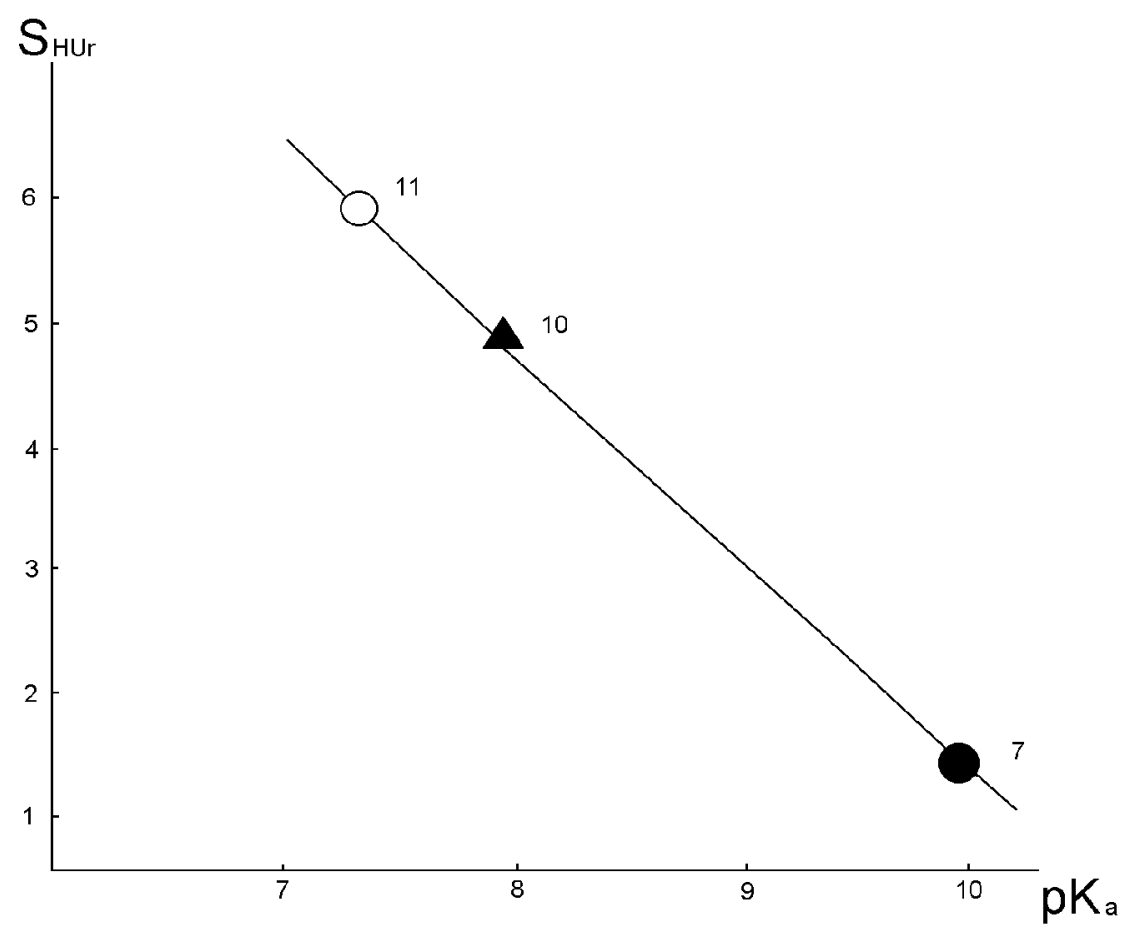

Figure. 2. Dependence of the solubility of uric acid stones on pKa of amines. The numbers of compounds in the figure and in the Table are the same

The increasing of $\mathrm{S}_{\mathrm{HUr}}$ with the increase of $\mathrm{pH}$ is the least for strong base PiPe $\left(\mathrm{pK}_{\mathrm{BH}}{ }^{+}=9.8\right)$. While for weak base Im substantial increasing equilibrium solubility of uric acid stones ( 2.5 order of magnitude) is characteristic. For a moderately strong base TEA intermediate increasing of $\mathrm{S}_{\mathrm{HUr}}$ is observed. We consider that different course of solubility curves for solutions of PiPe, TEA, and Im is conditioned, first of all, by changing relation of charged and neutral form of amine in the studied interval of $\mathrm{pH}$. Indeed, the computed dependence of concentration of charged particles in the solutions of amines on $\mathrm{pH}$ is analogous to the course of dependences in fig. 3 .

Analysis of fig. 1-3 permits us to make correct selection of the compound, its concentration and solution $\mathrm{pH}$ in order to provide desirable value of equilibrium solubility of uric acid stones in water solution. 


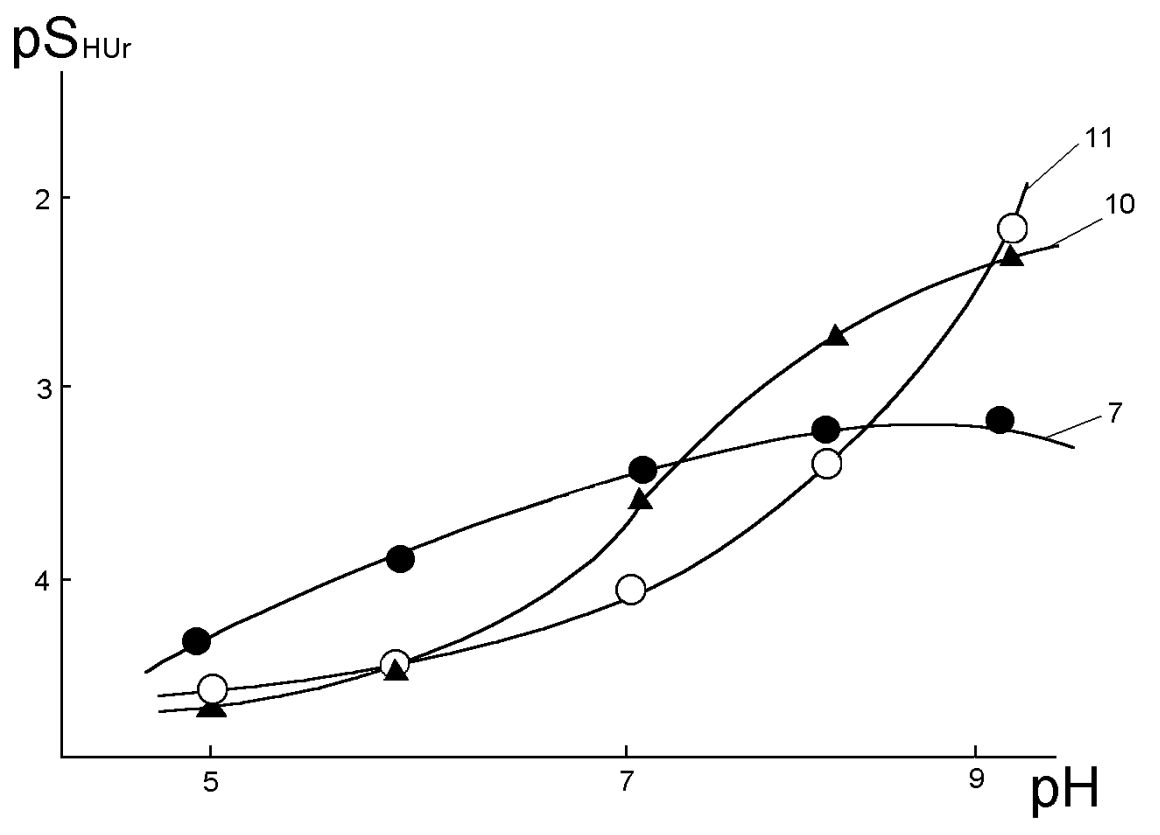

Figure 3. Dependence of logarithm of equilibrium solubility of uric acid stone on $\mathrm{pH}$ of the solution. The numbers of compounds in the figure and in the Table are the same

Previously it was established that, in solutions, which contain ions of ammonium, alkaline and alkaline-earth metals, the process of dissolution of uric acid concurs with the processes of sedimentation of hardly soluble urate salts on the concrement surface $[4,5]$. The hardest soluble urate is ammonium urate. Therefore, it is expected that the effect of amines on $\mathrm{S}_{\mathrm{HUr}}$ will be expressed rather originally in the presence of ammonium-ion. Indeed, at "normal" concentration of ammonium in the urine of healthy people (46 mM) we observed different dependences.

In fig. 4 the dependencies $\mathrm{S}_{\mathrm{HUr}}=\mathrm{f}\left(\left[\mathrm{NH}_{4}{ }^{+}\right]\right)$for $0.35 \mathrm{M}$ PiPe, TEA and $\mathrm{Im}$ at $\mathrm{pH}=8$ are shown. It is remarkable that the adding of ammonium in various concentrations, into the solution of weak base imidazole leads to manifold change of $S_{H U r}$. For moderate base TEA such effect is less expressed. And for solutions of strong base PiPe the wide varying of ammonium concentration does not effect on $\mathrm{S}_{\mathrm{HUr}}$ practically. $\mathrm{S}_{\mathrm{HUr}}$ value is a real constant and equals to $1 \mathrm{mM}(\sim 168 \mathrm{mg} / \mathrm{l})$. 


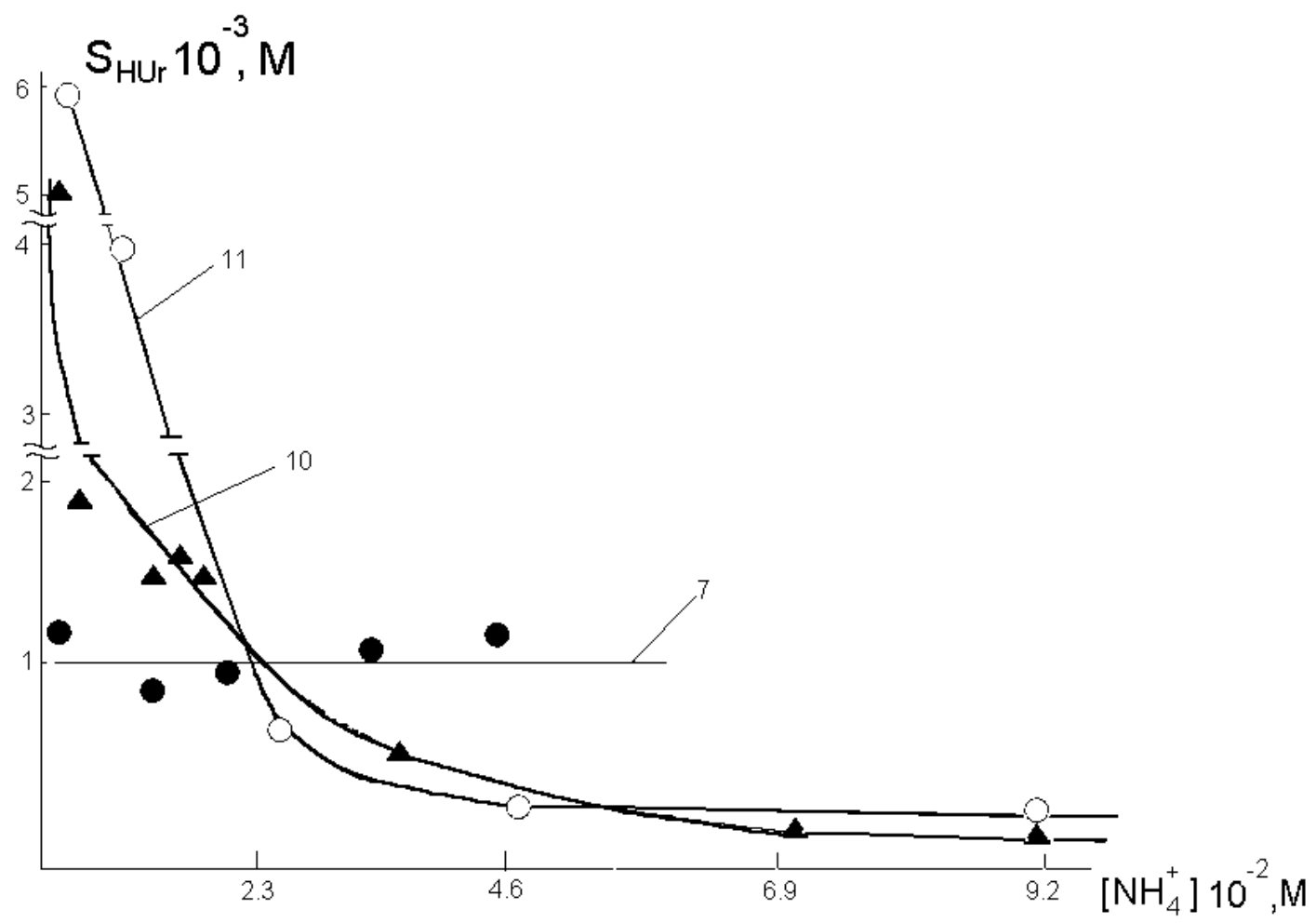

Figure 4. Dependence of equilibrium solubility of uric acid stone on ammonium concentration. The numbers of compounds in the figure and in the Table are the same

Analysis of the course of the dependences $\mathrm{S}_{\mathrm{HUr}}=\mathrm{f}\left(\left[\mathrm{NH}_{4}{ }^{+}\right]\right)$in fig. 4 may be used to select the amine to provide maximal solubility of uric acid stone at various ammonium concentration in solution. So, it follows that at $\left[\mathrm{NH}_{4}{ }^{+}\right]<0.5$ "norm", in solutions imidazole and triethanolamine are preferable for increasing the solubility of uric acid stones. But, when $\left[\mathrm{NH}_{4}{ }^{+}\right]>1.0$ "norm", piperazine is useful for this purpose. Mechanism of rather different action of these amines in the solubility of uric acid stones, apparently may be explained by following reasons.

Evidently, weak bases Im and TEA increase the solubility of HUr due to the formation of soft molecular complexes with HUr. Naturally, such complexes can not overcome the concurrence with thermodynamically favorable and hardly soluble ammonium urate. Therefore, weak amines may increase the solubility only in the presence of small quantities of ammonium in solution. If in the solution $\left[\mathrm{NH}_{4}^{+}\right]>0.5$ "norm", soft complexes of these weak bases can not prevent the formation of ammonium urate and its precipitation on the concrement surface.

In the solution of strong base PiPe, its urates form rather quickly. So, better 
solubility of piperazinium urates provides significantly better equilibrium solubility of HUr concrement independent of $\left[\mathrm{NH}_{4}^{+}\right]$all over the interval that was studied.

Equilibrium solubility of uric acid stone in cation containing solutions, simulating human urine was measured also. It was found that, at "normal" concentration of cations, the dissolution of stone was a stable process, occurring in a wide range of $[\mathrm{Am}]$ and in these conditions $\mathrm{S}_{\mathrm{HUr}}$ values were obtained for PiPe, TEA, and Imsystems: $2.3 \mathrm{mM}, 1.1 \mathrm{mM}$, and 2.4mM, corresp.

When comparing the course of dependence $\mathrm{S}_{\mathrm{HUr}}=\mathrm{f}\left(\left[\mathrm{NH}_{4}{ }^{+}\right]\right)$in fig-4 with the peculiarities of dissolution of uric acid stones in multicomponent salt systems, it is notable that availability of alkaline and alkaline-earth metal ions with simultaneous existence of ammonium ion leads to increase equilibrium solubility of uric acid stones. It is in complete conformity with previous results [1,5], in which it had been shown that, maximal $\mathrm{S}_{\mathrm{HUr}}$ in salt-solutions corresponds to "normal" concentration of cations in urine of healthy person. The role of amines in the systems, which were studied, is to increase supplementarily $\mathrm{S}_{\mathrm{HUr}}$ and to stabilize the process of dissolution of stones.

Conclusions. It was shown that "soft bases" increase the equilibrium solubility of uric acid stones and stabilize the process of litholysis. Here, depending on the concentration of ammonium ion and cations of alkaline and alkaline-earth metals, it is purposeful to use either weak bases imidazole and triethanolamine or more stronger base - piperazine.

\section{References.}

1. Bilobrov V, M, Bogdan N, M, Bilobrov S, V, Roy A: Influence of Cations and Total Protein of Urine on the Solubility and Probability of Urate Stone Formation in Kidneys. Urol. Internationalis 2002; 68, 118-121. doi: 10.1159/000048431

2. P.Porter. Physico-chemical Factors Involved in Urate Calculus Formation: I.—Solubility. Research in Veterinary Science. Volume 4, Issue 4, October 1963, Pages 580-591. https://doi.org/10.1016/S0034-5288(18)34844-6

3. Pearson R. G. Hard and soft acids and bases, HSAB, part 1: Fundamental principles // J. Chem. Education. 1968. 45 ( 9): 581. doi:10.1021/ed045p581.

4. Martillo MA, Nazzal L, Crittenden DB. The crystallization of monosodium urate. Curr. 
Rheumatol Rep. 2014 Feb;16(2):400. doi: 10.1007/s11926-013-0400-9.

5. Bilobrov VM, Litvinenko LM, Jedinyj JuG, et al. Dissolution of urate concrements. Urology and Nephrology (rus.) 1986, 2, 33-38 